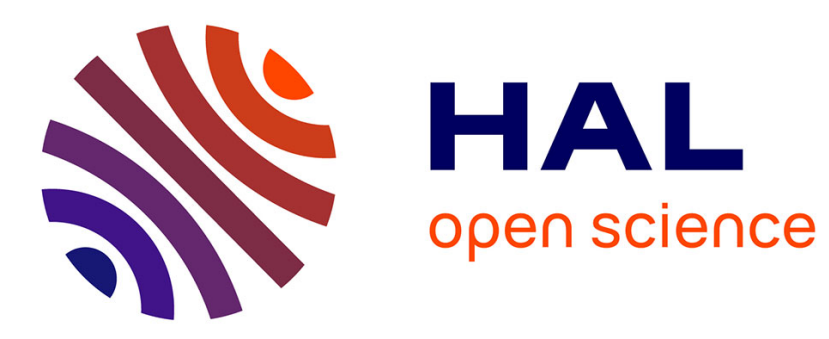

\title{
Diethyl Trichloromethylphosphonate
}

Angela Marinetti, Jean-François Betzer

\section{To cite this version:}

Angela Marinetti, Jean-François Betzer. Diethyl Trichloromethylphosphonate. Encyclopedia of Reagents for Organic Synthesis, 2014, 10.1002/047084289X.rn01731 . hal-02152308

\section{HAL Id: hal-02152308 \\ https://hal.science/hal-02152308}

Submitted on 11 Jun 2019

HAL is a multi-disciplinary open access archive for the deposit and dissemination of scientific research documents, whether they are published or not. The documents may come from teaching and research institutions in France or abroad, or from public or private research centers.
L'archive ouverte pluridisciplinaire HAL, est destinée au dépôt et à la diffusion de documents scientifiques de niveau recherche, publiés ou non, émanant des établissements d'enseignement et de recherche français ou étrangers, des laboratoires publics ou privés. 


\section{Diethyl Trichloromethylphosphonate}

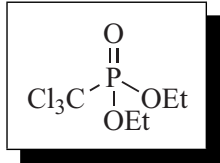$$
\text { [866-23-9] } \quad \mathrm{C}_{5} \mathrm{H}_{10} \mathrm{Cl}_{3} \mathrm{O}_{3} \mathrm{P} \quad \text { (MW 255.46) }
$$$$
\mathrm{InChI}=1 \mathrm{~S} / \mathrm{C} 5 \mathrm{H} 10 \mathrm{Cl} 3 \mathrm{O} 3 \mathrm{P} / \mathrm{c} 1-3-10-12(9,11-4-2) 5(6,7) 8 / \mathrm{h} 3-
$$$$
4 \mathrm{H} 2,1-2 \mathrm{H} 3
$$$$
\text { InChIKey }=\text { RVAQSYWDOSHWGP-UHFFFAOYSA-N }
$$

(Horner-Emmons reagent for the synthesis of 1,1-dichloro-1alkenes from carbonyl compounds; precursor of diethyl 1,1dichloro- $\alpha$-alkylmethylphosphonates)

Alternate Name: trichloromethylphosphonic acid diethyl ester. Physical Data: bp $127-128^{\circ} \mathrm{C} / 13 \mathrm{mmHg}{ }^{1} d 1.3622 \mathrm{~g} \mathrm{~cm}^{-3.2}$ $n^{25} \mathrm{D} 1.4582 .^{1}$

Solubility: sol common organic solvents.

Form Supplied in: colorless mobile liquid.

Analysis of Reagent Purity: by NMR $\left({ }^{31} \mathrm{P}\right.$ NMR: $6.5 \mathrm{ppm} ;{ }^{1} \mathrm{H}$ NMR). ${ }^{3}$

Preparative Methods: commercially available. It can be readily prepared via the Arbuzov reaction from triethyl phosphite and carbon tetrachloride. ${ }^{1}$

Handling, Storage, and Precautions: irritant; store under inert atmosphere at $4{ }^{\circ} \mathrm{C}$.

Reactions with Carbonyl Compounds: Aldehydes, Ketones, and Lactones. Diethyl trichloromethylphosphonate is used mainly in the synthesis of 1,1-dichloro-1-alkenes from carbonyl compounds via Horner-Wadsworth-Emmons (HWE)-type reactions. The reagent represents a convenient alternative to Wittigtype reagents, such as carbon tetrachloride/triphenylphosphine ${ }^{\mathbf{4 , 5}}$ or carbon tetrachloride/tris(dimethylamino)phosphine, ${ }^{\mathbf{6}}$ as far as it avoids formation of phosphine oxide by-products.

For these olefination reactions, the lithiated phosphonate is generated at very low temperatures (typically $-100^{\circ} \mathrm{C}$ ) by a chloride-lithium exchange: treatment of diethyl trichloromethylphosphonate with 1 equiv of $n$-butyllithium leads to the monolithio derivative $\mathrm{LiCCl}_{2} \mathrm{P}(\mathrm{O})(\mathrm{OEt})_{2}$, which then reacts with carbonyl compounds to afford 1,1-dichloro-1-alkenes. ${ }^{7}$ In the lithiation of $\mathrm{Cl}_{3} \mathrm{C}-\mathrm{P}(\mathrm{O})(\mathrm{OEt})_{2}$, an excess of $n$-butyllithium should be avoided, since polylithiation to give $\mathrm{Li}_{2} \mathrm{CClP}(\mathrm{O})(\mathrm{OEt})_{2}$ becomes possible. Initially, THF was used as the solvent, but the method could be later improved by using a 40:60 mixture of tetrahydrofuran/diethyl ether. ${ }^{8}$ Representative examples of the olefination reaction are given in eq 1 .

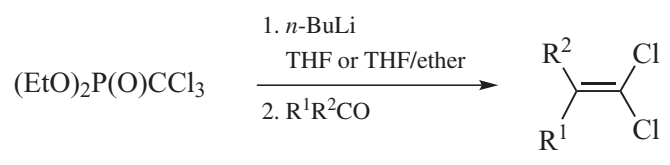

Some of the gem-dichloroolefins above have been used as substrates for the synthesis of terminal alkynes, as well as of chloroand alkyl-substituted alkynes. ${ }^{8}$
Additional examples of Wittig-Horner olefinations by means of diethyl trichloromethylphosphonate include the olefination of both lanosterol- ${ }^{\mathbf{1 3}}$ and carbohydrate-derived aldehydes. ${ }^{\mathbf{1 4}}$ Also, the method has been used to access highly functionalized intermediates in the synthesis of natural products and other complex molecules. For instance, the trans-decalin-derived aldehyde (eq 2) has been converted into the corresponding dichloroolefin, which is a key intermediate in the total synthesis of ingenol. ${ }^{\mathbf{1 5}, 16}$

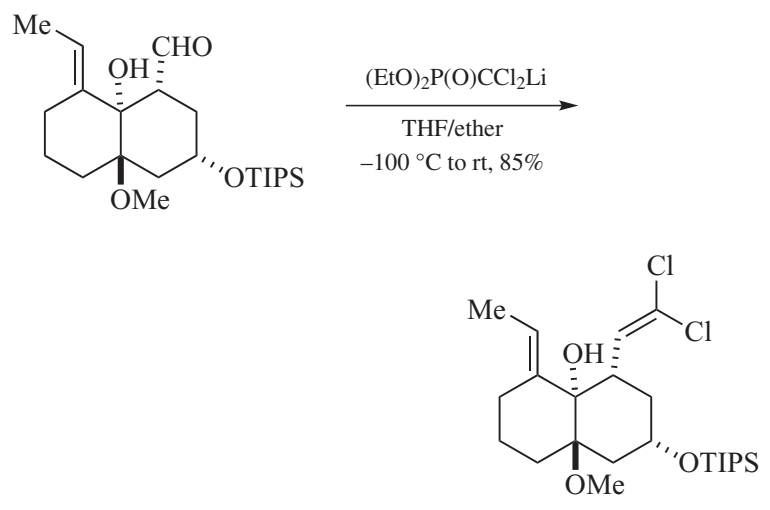

The same olefination method has been applied to the $\alpha, \beta$ unsaturated aldehyde (eq 3 ) for the synthesis of trans-kumausyne, a tetrahydrofuranoid lipid isolated from red algae, ${ }^{17}$ as well as to the geraniol-derived aldehyde (eq 4) for the synthesis of humulene, a sesquiterpene constituent of oil of hops. ${ }^{18}$<smiles></smiles><smiles>[Y15]O[C@H]1C[C@H](C([R])[SbH])O[C@@H]1C/C=C/C=C(Cl)Cl</smiles><smiles>CCOC(=O)OCC(C)=CCCC(C)=CCC(C)(C)C=C(Cl)Cl</smiles>

The lithiated trichloromethylphosphonate has been used to convert a [4.3.2]propellatriene-derived dialdehyde into the corresponding bis-dichloroolefin (eq 5). A large excess (10 equiv) of the lithiated phosphonate is required for this reaction. The resulting bis-olefin was then converted into a bis-alkyne, which was used to generate polyynes via the photochemical extrusion of indan from the tricyclic scaffold. ${ }^{19,20}$ 


\begin{tabular}{|c|c|c|c|c|}
\hline Carbonyl compound & Conditions & Product & Yield (\%) & Reference \\
\hline $\mathrm{Me}_{3} \mathrm{CCHO}$ & $\mathrm{THF},-80^{\circ} \mathrm{C} / \mathrm{rt}, 24 \mathrm{~h}$ & $\mathrm{Me}_{3} \mathrm{CCH}=\mathrm{CCl}_{2}$ & 55 & 7 \\
\hline $\mathrm{Et}_{2} \mathrm{CHCHO}$ & THF/ether $40: 60,-100^{\circ} \mathrm{C} /$ reflux, $1 \mathrm{~h}$ & $\mathrm{Et}_{2} \mathrm{CHCH}=\mathrm{CCl}_{2}$ & 82 & 8 \\
\hline $\mathrm{C}_{9} \mathrm{H}_{19} \mathrm{CHO}$ & THF/ether $40: 60,-100^{\circ} \mathrm{C} /$ reflux, $1 \mathrm{~h}$ & $\mathrm{C}_{9} \mathrm{H}_{19} \mathrm{CH}=\mathrm{CCl}_{2}$ & 92 & 8 \\
\hline $2-\mathrm{MeOC}_{6} \mathrm{H}_{5}-\mathrm{CHO}$ & THF/ether 40:60, $-100^{\circ} \mathrm{C} /$ reflux, $1 \mathrm{~h}$ & $2-\mathrm{MeOC}_{6} \mathrm{H}_{5}-\mathrm{CH}=\mathrm{CCl}_{2}$ & 94 & 8 \\
\hline \multirow[t]{2}{*}{$\mathrm{PhCH}=\mathrm{CH}-\mathrm{CHO}$} & THF/ether $40: 60,-100{ }^{\circ} \mathrm{C} /$ reflux, $1 \mathrm{~h}$ & $\mathrm{PhCH}=\mathrm{CH}-\mathrm{CH}=\mathrm{CCl}_{2}$ & 92 & 8 \\
\hline & THF/ether $40: 60,-100^{\circ} \mathrm{C} / \mathrm{rt}$, overnight & & 74 & 9 \\
\hline & $\mathrm{THF},-100^{\circ} \mathrm{C} / \mathrm{rt}$, overnight & & 78 & 10 \\
\hline $\mathrm{Me}_{2} \mathrm{CO}$ & THF, $-80^{\circ} \mathrm{C} /$ reflux, $1 \mathrm{~h}$ & $\mathrm{Me}_{2} \mathrm{C}=\mathrm{CCl}_{2}$ & 47 & 7 \\
\hline \multirow[t]{2}{*}{$\mathrm{Ph}_{2} \mathrm{CO}$} & $\mathrm{THF},-80^{\circ} \mathrm{C} /$ reflux, $1 \mathrm{~h}$ & $\mathrm{Ph}_{2} \mathrm{C}=\mathrm{CCl}_{2}$ & 69 & 7 \\
\hline & THF/ether $40: 60,-100^{\circ} \mathrm{C} /$ reflux, $1 \mathrm{~h}$ & & 90 & 8 \\
\hline & THF/ether $40: 60,-100^{\circ} \mathrm{C} /$ reflux, $1 \mathrm{~h}$ & & 69 & 11 \\
\hline & THF/ether, $-115^{\circ} \mathrm{C} /$ reflux, $18 \mathrm{~h}$ & & 79 & 12 \\
\hline
\end{tabular}

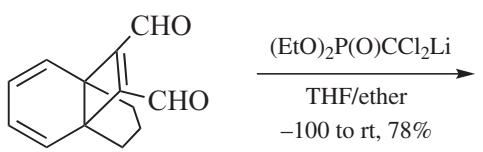<smiles>ClC(Cl)=CC1CC2(C=CC=CC2)C1C=C(Cl)Cl</smiles>

In addition to aldehydes and ketones, lithiated trichloromethylphosphonate can be made to react also with lactones (eq 6). The usual dichloroolefination protocol affords the desired dichloromethylene bicyclo[2.2.2] octane derivative in acceptable yield $(58 \%)^{21}$<smiles>O=C1OC2C=CC1C2</smiles>

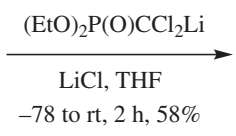<smiles>ClC(Cl)=C1OC2C=CC1C2</smiles>

The diethyl dichloromethylphosphonate carbanion can also be generated by electrochemical reduction of trichloromethylphosphonate. It then reacts with carbonyl compounds, in aprotic media, to afford 1,1-dichloro-1-alkenes, ${ }^{\mathbf{2 2}-\mathbf{2 5}}$ while, in protic media, it affords dichloromethylphosphonate quantitatively. The electrochemical method has the advantage of avoiding the use of strongly basic alkyllithium reagents, but the yields are generally lower.
Synthesis of $\alpha$-Substituted Phosphonates from Trichloromethylphosphonate and Alkyl Halides. Diethyl trichloromethylphosphonate gives access to diethyl 1,1-dichloro- $\alpha$-alkylmethylphosphonates via alkylation of the corresponding anion $\mathrm{LiCCl}_{2} \mathrm{P}(\mathrm{O})(\mathrm{OEt})_{2}$ with $n$-alkyl bromides or iodides (eq 7). ${ }^{\mathbf{7 , 2 6}}$ Alternatively, dichloro- $\alpha$-alkylmethylphosphonates can be prepared from trichloromethylphosphonates via a two-step procedure. The first step is the reduction of diethyl trichloromethylphosphonate by treatment with iso-propylmagnesium chloride and subsequent hydrolysis with hydrogen chloride (ethanol solution) at low temperatures (eq 7). ${ }^{27,28}$ Then, alkylation of $\mathrm{HCCl}_{2} \mathrm{P}(\mathrm{O})$ $(\mathrm{OEt})_{2}$ is carried out under internal quenching conditions, in order to trap the unstable lithiated phosphonate $\mathrm{LiCCl}_{2} \mathrm{P}(\mathrm{O})(\mathrm{OEt})_{2}$ and to prevent formation of the carbene via $\mathrm{LiCl}$ elimination. With this procedure, the yields are often higher than those obtained by the direct metalation of trichloromethylphosphonate.

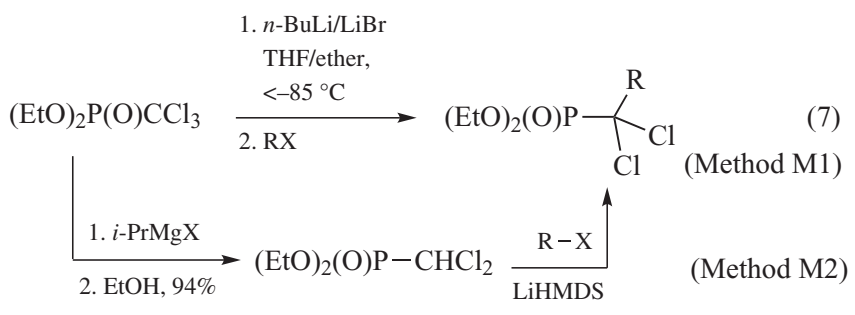




\begin{tabular}{lcc}
\hline \multicolumn{1}{c}{$\mathrm{RX}$} & Yield (\%) & Reference \\
\hline $\mathrm{H}_{2} \mathrm{C}=\mathrm{CHCH}_{2} \mathrm{Br}$ & $77(\mathrm{M} 1)$ & 7 \\
& $67(\mathrm{M} 2)$ & 27 \\
$\mathrm{MeCH}=\mathrm{CHCH}_{2} \mathrm{Br}$ & $75(\mathrm{M} 1)$ & 26 \\
& $87(\mathrm{M} 2)$ & 27 \\
$\mathrm{MeBr}^{\mathrm{a}}$ & $81(\mathrm{M} 1)$ & 26 \\
$n-\mathrm{PrI}$ & $76(\mathrm{M} 1)$ & 26 \\
& $88(\mathrm{M} 2)$ & 27 \\
$\mathrm{PhCH}_{2} \mathrm{Br}$ & $92(\mathrm{M} 2)$ & 27 \\
\hline
\end{tabular}

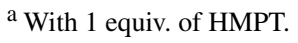

Notably, alkylation of $\mathrm{LiCCl}_{2} \mathrm{P}(\mathrm{O})(\mathrm{OEt})_{2}$ with 4,6-heptadienylbromide has been used as the first step in a reaction sequence leading to the phosphabicyclo[4.3.0]non-4-ene derivative (eq 8). ${ }^{\mathbf{2 9}}$

$$
\begin{aligned}
& (\mathrm{EtO})_{2} \mathrm{P}(\mathrm{O}) \mathrm{CCl}_{3} \underset{\text { 2. }}{\stackrel{1 . n \text {-BuLi, THF, }-85^{\circ} \mathrm{C}}{\mathrm{Br}}} \\
& \underbrace{\mathrm{Cl}}_{85 \%}
\end{aligned}
$$

Preparation and Synthetic Applications of Lithio(trimethylsilyl)chloromethylphosphonate. The monolithio derivative $\mathrm{LiCCl}_{2} \mathrm{P}(\mathrm{O})(\mathrm{OEt})_{2}$ can be trapped with chlorotrimethylsilane to afford diethyl dichloro(trimethylsilyl)methylphosphonate in $70 \%$ yield (eq 9). ${ }^{7}$ On the other hand, treatment of a mixture of diethyl trichloromethylphosphonate and trimethylsilyl chloride with 2 equiv of $n$-butyllithium leads to a stable $\alpha$-silylated $\alpha$-phosphonylated carbanion. This species is formed in quantitative yield and can be handled at $0{ }^{\circ} \mathrm{C}$ without decomposition or rearrangement (eq 10). ${ }^{\mathbf{3 0}}$
This silylated carbanion proved to be a very useful intermediate, being trapped with a variety of electrophilic reagents. For instance, trapping with aromatic or aliphatic aldehydes results in a Peterson-like olefination reaction, giving mixtures of isomeric $\alpha$-chlorovinylphosphonates (eq 11). ${ }^{\mathbf{3 0}}$ Further dehydrochlorination of the chlorovinylphosphonates with LiHMDS affords the corresponding 1-alkynylphosphonates. ${ }^{31}$<smiles>CCOC(=O)OCC</smiles>

The same silylated intermediate can be alkylated with alkyl bromides or iodides (MeI, 91\%; $n$-BuI, $81 \% ; \mathrm{H}_{2} \mathrm{C}=\mathrm{CH}-\mathrm{CH}_{2} \mathrm{Br}$, $\left.87 \%, \mathrm{PhCH}_{2} \mathrm{Br}, 80 \%\right) .{ }^{30}$ Notably, the double alkylation with $\gamma / \omega$ dibromoalkanes, in a two-step sequence, gives access to mediumsize cycloalkylphosphonates (four- to seven-membered rings, $18-70 \%$ yields) from which the silyl group can be removed by treatment with TBAF (eq 12). ${ }^{\mathbf{3 2}}$

$\alpha$-Alkyl- $\alpha$-silyl-chloromethylphosphonates obtained from the silylated carbanion can be involved in subsequent reactions with ethyl formate to afford 1-formylalkylphosphonates in good yields (eq 13). ${ }^{33}$

Finally, the stabilized carbanion can be quenched with deuterium oxide. Subsequent treatment of the silylated derivative with LiOD allow incorporation of a second deuterium atom, by cleavage of the $\mathrm{C}-\mathrm{Si}$ bond, to afford $\alpha, \alpha$-dideuterio-chloromethylphosphonate (eq 14). Analogous deuteration procedures have been applied also to $\alpha$-alkyl- $\alpha$-silyl-chloromethylphosphonates to generate $\alpha, \alpha$-dideuterio-alkylphosphonates that are important intermediates for the synthesis of deuterated olefins by HornerEmmons olefination. ${ }^{\mathbf{3 0 , 3 4}}$

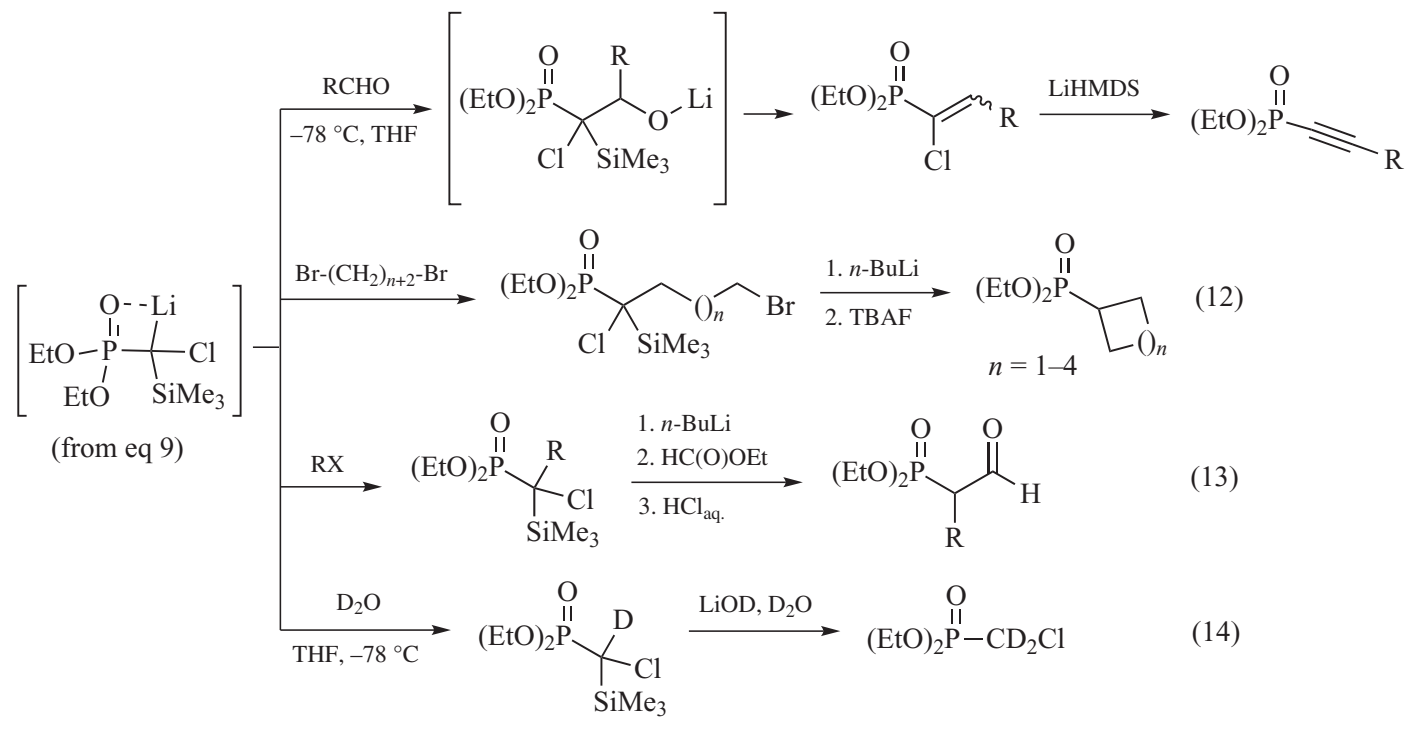




\section{Miscellaneous}

Metal-catalyzed Insertions of Olefins in the C-Cl Bond. Diethyl trichloromethylphosphonate reacts with olefins under $\mathrm{Cu}(\mathrm{I})$ or $\mathrm{Fe}$ (III) catalysis to give insertion of the olefinic substrate into one of the $\mathrm{C}-\mathrm{Cl}$ bonds. This radical process can be performed with either $\mathrm{FeCl}_{3}$ in the presence of triethylammonium chloride, ${ }^{\mathbf{3 5}}$ $\mathrm{CuCl} / i \mathrm{BuNH}_{2}$, or $\mathrm{CuCl} /$ phenanthroline (eq 15). ${ }^{\mathbf{3 6}}$

$$
(\mathrm{EtO})_{2} \mathrm{P}(\mathrm{O}) \mathrm{CCl}_{3}+\mho^{\mathrm{R}} \stackrel{\text { Cat. }}{\longrightarrow}(\mathrm{EtO})_{2}{ }_{\mathrm{Cl}}^{\mathrm{P}} \overbrace{\mathrm{Cl}}^{\mathrm{O}} \mathrm{Cl}_{\mathrm{Cl}}^{\mathrm{R}}
$$

\begin{tabular}{lccc}
\hline \multicolumn{1}{c}{$\mathrm{RX}$} & \multicolumn{2}{c}{ Catalyst/conditions } & \multicolumn{2}{c}{ Yield } \\
$(\%)$ & Reference \\
\hline $\mathrm{H}_{2} \mathrm{C}=\mathrm{CH}-\mathrm{Et}$ & $\mathrm{FeCl}_{3} / \mathrm{Et}_{3} \mathrm{NHCl}_{110}{ }^{\circ} \mathrm{C}, 9 \mathrm{~h}$ & 62 & 35 \\
$\mathrm{H}_{2} \mathrm{C}=\mathrm{CH}-$ & $\mathrm{CuCl} / i-\mathrm{BuNH}_{2} 100^{\circ} \mathrm{C}, 12 \mathrm{~h}$ & 59 & 36 \\
$\mathrm{CO}_{2} \mathrm{Et}$ & & & \\
$\mathrm{H}_{2} \mathrm{C}=\mathrm{CH}-\mathrm{Ph}$ & $\mathrm{CuCl} / i-\mathrm{BuNH}_{2} 100^{\circ} \mathrm{C}, 12 \mathrm{~h}$ & 64 & 36 \\
$\mathrm{H}_{2} \mathrm{C}=\mathrm{CH}-\mathrm{Bu}$ & $\mathrm{CuCl} / i-\mathrm{BuNH}_{2} 100^{\circ} \mathrm{C}, 12 \mathrm{~h}$ & 55 & 36 \\
\hline
\end{tabular}

Alkylation by Tributylborane. Diethyl trichloromethylphosphonate has been used as a carbenoid precursor in the reaction with $\mathrm{Bu}_{3} \mathrm{~B}$ (eq 16). ${ }^{3}$ The process involves formal insertion of the carbenoid intermediate $(\mathrm{EtO})_{2} \mathrm{P}(\mathrm{O}) \mathrm{CCl} \cdot \mathrm{ClLi}$ in the $\mathrm{B}-\mathrm{Bu}$ bond and subsequent hydrolysis, leading to the removal of the boron substituent. In this way, a butyl-substituted chloromethylphosphonate is obtained in good yield.

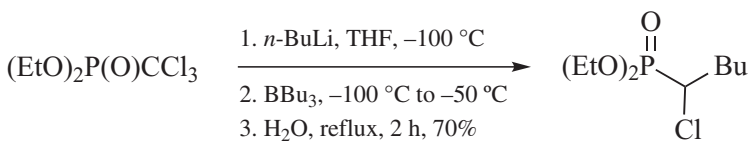

1. Kosolapoff, G. M., J. Am. Chem. Soc. 1947, 69, 1002.

2. Burger, L. L.; Wagner, R. M., Ind. Eng. Chem. Chem. Eng. Data Series 1958, 3, 310 .

3. Antczak, M. I.; Montchamp, J.-L., Org. Lett. 2008, 10, 977.

4. Speziale, A. J.; Marco, G. J.; Ratts, K. W., J. Am. Chem. Soc. 1960, 82, 1260.

5. Rabinowitz, R.; Marcus, R., J. Am. Chem. Soc. 1962, 84, 1312.
6. Combret, J.-C.; Villieras, J.; Lavielle, G., Tetrahedron Lett. 1971, 12, 1035 .

7. Seyferth, D.; Marmor, R. S., J. Organomet. Chem. 1973, 59, 237.

8. Villieras, J.; Perriot, P.; Normant, J.-F., Synthesis 1975, 458.

9. Iyoda, M.; Nakagawa, T.; Ebe, H.; Oda, M.; Nakagawa, M.; Yamamoto, K.; Higuchi, H.; Ojima, J., Bull. Chem. Soc. Jpn 1994, 67, 778.

10. Arthuis, M.; Lecup, A.; Roulland, E., Chem. Commun. 2010, 46, 7810.

11. Roedig, A.; Ganns, E. M.; Henrich, C.; Schnutenhaus, H., Liebigs Ann. Chem. 1981, 1674.

12. Cordes, J.; Wessel, C.; Harms, K.; Koert, U., Synthesis 2008, 2217.

13. Chung, S.-K.; Ryoo, C. H.; Yang, H. W.; Shim, J.-Y.; Kang, M. G.; Lee, K. W.; Kang, H. I., Tetrahedron 1998, 54, 15899.

14. Ferrier, R. J.; Tyler, P. C., Carbohydr. Res. 1985, 136, 249.

15. Nakamura, T.; Matsui, T.; Tanino, K.; Kuwajima, I., J. Org. Chem. 1997, 62,3032

16. Tanino, K.; Onuki, K.; Asano, K.; Miyashita, M.; Nakamura, T.; Takahashi, Y.; Kuwajima, I., J. Am. Chem. Soc. 2003, 125, 1498.

17. Brown, M. J.; Harrison, T.; Overman, L. E., J. Am. Chem. Soc. 1991, $113,5378$.

18. Miyaura, N.; Suginome, H.; Suzuki, A., Tetrahedron Lett. 1984, 25, 761.

19. Tobe, Y.; Fujii, T.; Naemura, K., J. Org. Chem. 1994, 59, 1236.

20. Tobe, Y.; Fujii, T.; Matsumoto, H.; Tsumuraya, K.; Noguchi, D.; Nakagawa, N.; Sonoda, M.; Naemura, K.; Achiba, Y.; Wakabayashi, T., J. Am. Chem. Soc. 2000, 122, 1762.

21. Robertson, J.; Fowler, T. G., Org. Biomol. Chem. 2006, 4, 4307.

22. Tue Bi, B.; Devaud, M., Tetrahedron Lett. 1987, 28, 3799.

23. Karrenbrock, F.; Schäfer, H. J.; Langer, I., Tetrahedron Lett. 1979, 20, 2915.

24. Le Menn, J.-C.; Sarrazin, J.; Tallec, A., Can. J. Chem. 1989, 67, 1332.

25. van Tilborg, W. J. M.; Smit, C. J., J. Recl. Trav. Chim. Pays-Bas 1980, 99, 202.

26. Coutrot, P.; Laurenco, C.; Normant, J.-F.; Perriot, P.; Savignac, P.; Villieras, J., Synthesis 1977, 615.

27. Carran, J.; Waschbüsch, R.; Marinetti, A.; Savignac, P., Synthesis 1996, 1494.

28. Marinetti, A.; Savignac, P., Org. Synth. 1997, 74, 108.

29. Pilard, J.-F.; Gaumont, A.-C.; Friot, C.; Denis, J.-M., Chem. Commun. 1998, 457.

30. Teulade, M.-P.; Savignac, P., J. Organomet. Chem. 1988, 338, 295.

31. Dizière, R.; Savignac, P., Tetrahedron Lett. 1996, 37, 1783.

32. Grandin, C.; Collignon, N.; Savignac, P., Synthesis 1995, 239.

33. Zanella, Y.; Berté-Verrando, S.; Diziere, R.; Savignac, P., J. Chem. Soc., Perkin Trans. 1 1995, 2835.

34. Berté-Verrando, S.; Nief, F.; Patois, C.; Savignac, P., J. Chem. Soc., Perkin Trans. 1 1994, 821.

35. Rosin, H.; Asscher, M., J. Org. Chem. 1975, 40, 3298.

36. Villemin, D.; Sauvaget, F.; Hàjek, M., Tetrahedron Lett. 1994, 35, 3537.

Angela Marinetti \& Jean-François Betzer Institut de Chimie des Substances Naturelles, CNRS UPR 2301, 91198 Gif-sur-Yvette, France 